\title{
Quantifying scattering coefficient for multiple scattering effect by combining optical coherence tomography with finite-difference time-domain simulation method
}

Ling-Hsuan Tsai

Po Nien Yang

Chung-Chih Wu Hoang Yan Lin 


\title{
Quantifying scattering coefficient for multiple scattering effect by combining optical coherence tomography with finite-difference time-domain simulation method
}

\author{
Ling-Hsuan Tsai, ${ }^{a}$ Po Nien Yang, ${ }^{a}$ Chung-Chih $\mathrm{Wu}^{\mathrm{a}, \mathrm{b}}$ and Hoang Yan Lin ${ }^{\mathrm{a}, *}$ \\ ${ }^{a}$ National Taiwan University, Graduate Institute of Photonics and Optoelectronics, Department of Electrical Engineering, Taipei, Taiwan \\ ${ }^{b}$ National Taiwan University, Graduate Institute of Electronics Engineering, Department of Electrical Engineering, Taipei, Taiwan
}

\begin{abstract}
In optical coherence tomography (OCT) systems, to precisely obtain the scattering properties of samples is an essential issue in diagnostic applications. Especially with a higher density turbid medium, the light interferes among the adjacent scatters. Combining an OCT experiment with the finite-difference time-domain simulation method, the multiple scattering effect is shown to affect the scattering properties of medium depending on the interparticle spacing. The far-field scattering phase function of scatters with various diameters was simulated to further analyze the corresponding anisotropy factors, which can be introduced into the extended Huygens-Fresnel theory to find the scattering coefficient of measured samples. ๑ 2018 Society of Photo-Optical Instrumentation Engineers (SPIE) [DOI: 10.1117/1.JBO.23.8.086004]
\end{abstract}

Keywords: optical coherence tomography; multiple scattering effect; finite-difference time-domain method; extended HuygensFresnel theory.

Paper 180209R received Apr. 15, 2018; accepted for publication Aug. 3, 2018; published online Aug. 28, 2018.

\section{Introduction}

Optical coherence tomography (OCT) is a potentially noninvasive sensing optical imaging system for various clinical diagnosis applications, and has a lot of developments particularly in the past decade. ${ }^{1,2}$ As an emerging imaging system, OCT can provide high-resolution axial structure images inside tissue from two- (A-scan) to three-dimensional (A-scan and B-scan). ${ }^{3}$ It is an interferometry-based optical technique, collecting the backscattered light from the sample to further extract the optical characteristic details within the tissue. Currently in this field, precise analysis of optical properties from the inhomogeneous and dense tissue remains challenging work. ${ }^{4}$ Different models of OCT have been established to obtain the optical properties of samples with further consideration of multiple scattering effect. ${ }^{5,6}$ Among these works, Thrane et al. were the first to propose the use of modulated interferometer signals of an OCT system, which can be expressed analytically based on the extended Huygens-Fresnel (EHF) principle, and to further consider the effect of multiple scattering. ${ }^{7}$ In addition, EHF theory has commonly been utilized to numerically quantify optical properties from A-scan signals of OCT images. However, in the EHF theory, there are two main flexible parameters highly correlated with the slope of OCT signals, i.e., the scattering coefficient $\left(\mu_{\mathrm{s}}\right)$ and the anisotropy factor $(g)$. Koach et al. ${ }^{8}$ reported in 2011 that the scattering phase function in a backward direction could be determined as the ratio of the backscattering coefficient to the scattering coefficient (only single scattering) detected from the CCD, but without the consideration of multiple scatterings. Then in 2013, Nguyen et al. ${ }^{9}$ further improved the OCT system by introducing a new setup of the transmission OCT system, in

*Address all correspondence to: Hoang Yan Lin, E-mail: hoangyanlin@ ntu.edu tw order to detect transmitted signals, and they introduced the structure factor (Percus-Yevick theory) to show the concentration-dependent scattering coefficients. In 2015, Almasian et al. ${ }^{10,11}$ proposed that a concentration-dependent scattering coefficient of the sample could be obtained from the Mie theory by incorporating the structure factor of Percus-Yevick theory in the calculation procedure. In this study, we propose a new analysis flow by combining the OCT system with the finite-difference-timedomain (FDTD) simulation method, to quantify the scattering coefficient of mesoporous $\mathrm{TiO}_{2}$ beads after introducing $g_{\text {dep }}$ factors into EHF theory. The benefit of combining with the FDTD method is that the characteristics of objects can be flexibly modified, including the shape (not only sphere), the refractive index, and the arrangement for inhomogeneous morphology. For comparison to the Percus-Yevick theory mentioned above, the structure factor can only hypothesize for a set of two hard spheres with fixed diameter size at a specific condition. ${ }^{12}$ In addition, differing from the algorithm of calculating the radial correlation function (radial distribution function) between adjacent particles in $\mathrm{P}-\mathrm{Y}$ theory, the near-field electrical field of the FDTD method can briefly demonstrate and show the light interaction when the object is scattered by incident light, and the far-field scattering pattern can be further determined.

First, the anisotropy factor $g_{\text {dep }}$ represents the intrinsic scattering characteristic properties (angular scattering pattern). The multiple scattering phenomena depending on the interparticle spacing (IPS) will be discussed in Sec. 3. From this method, the scattering phase function $\left[p_{\text {dep }}(\theta)\right]$ can be simulated to predict the corresponding anisotropy factor $g_{\text {dep }}$ of mesoporous $\mathrm{TiO}_{2}$ beads samples with different diameters varied from 20, 150,300 , to $500 \mathrm{~nm}$, respectively. In Sec. 4, the A-scan profiles

$1083-3668 / 2018 / \$ 25.00$ @) 2018 SPIE 
(a)

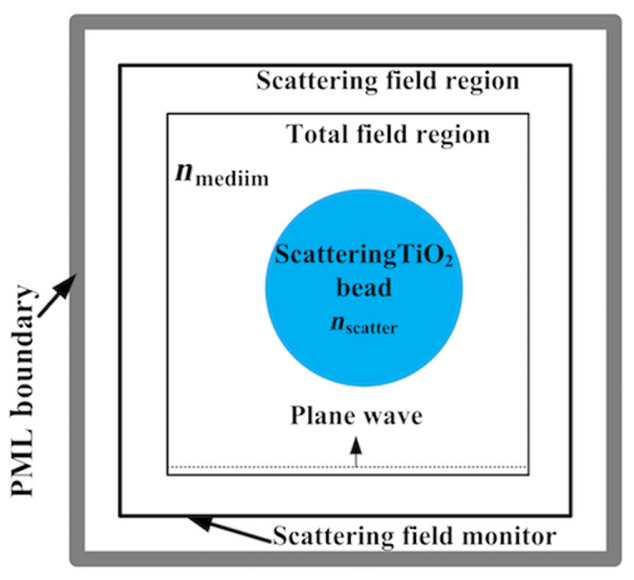

(b)

\section{The schematic of $\mathrm{TiO}_{2}$ sample with various diameters in a unit cell}

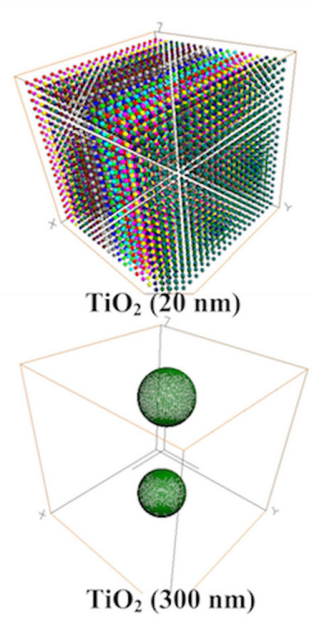

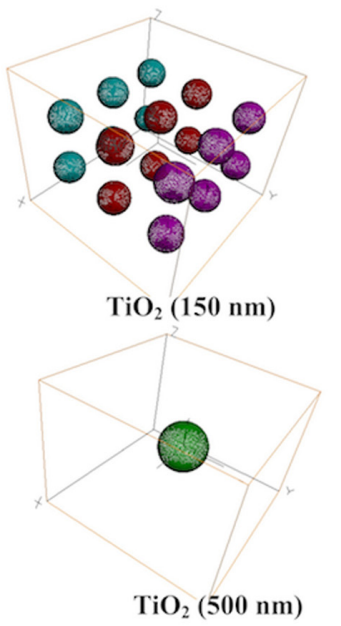

Fig. 1 (a) General setup of FDTD simulation for computing the phenomenon of light scattering effect. (b) The schematic diagram of $\mathrm{TiO}_{2}$ for different diameters embedded in a unit cell $\left(\# / \mathrm{m}^{3}\right)$.

are extracted by signal processing procedures from the interference patterns and then fitted by the EHF theory with consideration for the corresponding anisotropy factor $g_{\text {dep }}$, which is introduced to the EHF model to fit the scattering coefficient of each mesoporous $\mathrm{TiO}_{2}$ bead sample. Also, different calculations of the anisotropy factor $g_{\text {dep }}$ between the Mie scattering model and the FDTD method are compared.

\section{Methods and Experimental Setup}

\subsection{Finite-Difference-Time-Domain Method}

Since the development of the FDTD method, ${ }^{13}$ it has been utilized to solve Maxwell's equation numerically in the time domain to describe the complex behavior of light interaction and widely applied in solving the scattering issue in biological fields. ${ }^{14-16}$ In this study, we have utilized the R-Soft software with fullwave mode based on the FDTD algorithm. The FDTD algorithm is the computation process for implementing time and space derivatives of the electric and magnetic fields in Yee's cell. ${ }^{17}$ Solve the Maxwell's curl equations by first discretizing the equations with central differences in time and space and these equations can be further numerically solved by the software. To estimate the optical scattering properties of $\mathrm{TiO}_{2}$ beads with different diameters and embedded in thin-films, we align the scattering direction along with the line connecting the $\mathrm{TiO}_{2}$ beads, and they are separated by the IPS, which varies from 0 to $1000 \mathrm{~nm}$ in an interval of $250 \mathrm{~nm}$. The model used for FDTD is shown in Fig. 1(a): the wavelength of the incident plane wave is $\lambda_{0}=853 \mathrm{~nm}$, while the refractive index of mesoporous $\mathrm{TiO}_{2}$ beads is $n=2.5^{18,19}$ and assumed to be homogeneous and isotropic in distribution. The polarization of the incident wave is set as TE polarized. The grid size of calculation is set as $0.005 \mathrm{~nm}$, time step is $0.005 \mathrm{~s}$, and the incident wave is a plane wave in the enclosed area, respectively. With a perfectly matched layer (PML), it is composed of several grid points as the edge of the domain for the enclosed area, which can absorb the incident lights and result in no reflection.

In order to obtain the far-field scattering patterns, the virtual box with appropriate PML boundary is set to enclose the scattering objects (mesoporous $\mathrm{TiO}_{2}$ beads) and launch a plane wave inside an enclosed area, which is shown in Fig. 1(a). In the meantime, there are only scattering fields from objects propagating beyond the enclosed launch boundary, and unscattered light will be absorbed at the PML boundary. For obtaining the scattering phase function, the corresponding far field can be calculated using the near-field to far-field transformation from the simulation data in software. ${ }^{20}$

\subsection{Preparation of Mesoporous $\mathrm{TiO}_{2}$ Beads Samples}

Mesoporous $\mathrm{TiO}_{2}$ bead samples with average diameters $(D)$ of $20,150,300$, and $500 \mathrm{~nm}$ were prepared by the sol-gel process. Initially, the $\mathrm{TiO}_{2}$ powder weight percentage is $13 \mathrm{wt}$ \% (US Research Nanomaterials, Inc.) mixed with different percentages of isopropyl alcohol and DI water. Adding polyethylene glycol to the mixture, the $\mathrm{TiO}_{2}$ pastes were obtained after more than 1 week of stirring, and the $\mathrm{TiO}_{2}$ pastes prepared on glass substrates were heated by an oven at $450^{\circ} \mathrm{C}$ for $30 \mathrm{~min}$ to evaporate the solvent. In the process, the $\mathrm{TiO}_{2}$ were assumed to be homogeneously distributed. The final volume fraction of $\mathrm{TiO}_{2}$ bead samples was fixed as 0.03 for $\mathrm{TiO}_{2}$ beads with diameters varied from 20 to $500 \mathrm{~nm}$, respectively. Afterward, the samples of mesoporous $\mathrm{TiO}_{2}$ bead films on glass substrates were obtained.

For a suspension of colloidal system, the samples of $\mathrm{TiO}_{2}$ beads with volume fraction of 0.03 for different diameters are assumed to be homogeneously distributed; the schematic diagram of $\mathrm{TiO}_{2}$ beads in a unit cell $\left(1 \mu \mathrm{m}^{3}\right)$ for different diameters from 20 to $500 \mathrm{~nm}$ is shown in Fig. 1(b). The IPS parameter IPS $=2 r\left[\left(\frac{\phi_{m}}{\phi}\right)^{\frac{1}{3}}-1\right]$ is defined as the distance from the surface of one particle to the other [the line of connection between the two through the center of each bead, as shown in Fig. 4(a)], which changes according to the volume fraction in the colloidal system. ${ }^{21}$ The maximum volume fraction is $\phi_{m}$ with $\mathrm{TiO}_{2}$ samples, while $\varphi$ is the volume fraction of $\mathrm{TiO}_{2}$ bead samples, and $r$ is the radius of $\mathrm{TiO}_{2}$ beads. With the volume fraction of 0.03 , the values of IPS are 25,187 , 373 , and $623 \mathrm{~nm}$ for select $\mathrm{TiO}_{2}$ diameters between 20 and $500 \mathrm{~nm}$. 


\subsection{Extended Huygens-Fresnel Theory (Multiple Scattering) and Signal Analysis}

First, as an assistant method, the FDTD simulation process was utilized to calculate the electromagnetic near field and then transformed to a far-field scattering pattern, which is also called the phase function $p_{\text {dep }}(\theta)$, for different IPS between mesoporous $\mathrm{TiO}_{2}$ beads with different diameters from 20,150, 300, and $500 \mathrm{~nm}$. Here, the far-field $p_{\text {dep }}(\theta)$ is indicated as the probability density function of photons for every scattering angle $(\theta),{ }^{22}$ and the anisotropy factor $g_{\text {dep }}$ is the integral of phase function $p_{\text {dep }}(\theta)$ over all scattering angles, which can be expressed as

$g_{\text {dep }}=2 \pi \int_{0}^{\pi} p_{\text {dep }}(\theta) \cos (\theta) \sin (\theta) \mathrm{d} \theta=\left\langle\cos \theta_{\text {dep }}\right\rangle$,

where

$$
\int_{0}^{\pi} p_{\mathrm{dep}}(\theta) 2 \pi \sin (\theta) \mathrm{d} \theta=1 .
$$

Second, based on EHF theory, the amplitude of experimental OCT signals versus depth $z$ can be modulated as the function of two main flexible parameters for $g$ and $\mu_{\mathrm{s}}$. Once the multiple scattering effect appears, the right-hand side of point spread function (PSF) can be changed by slope variation at a larger axial depth as compared to that of a signal with only single scattering effect. ${ }^{23}$ In practice, the most common method to date to obtain the optical properties has been to analyze the axial A-scan signals based on EHF model at the right-hand side of PSF with increasing depth. Except for the fixed optical system parameters, the resulting heterodyne signals $\left\langle S^{2}(z)\right\rangle$ [Eqs. (3) and (4)] in depth can be mainly modulated by two competing factors, the anisotropy factor $\left(g=\left\langle\cos \theta_{\mathrm{rms}}\right\rangle, \theta_{\mathrm{rms}}\right.$ is average scattering angle) and the scattering coefficients $\left(\mu_{\mathrm{s}}\right){ }^{23}$ In addition, the fixed parameters in the system include the power $P_{\mathrm{R}}$ and $P_{\mathrm{S}}$ measured at the reference and sample arms; the quantum conversion efficiency $\alpha$ of the CCD; the backscattering cross-section $\alpha_{\mathrm{b}}$, and the $1 / e$ irradiance radii $w_{H}$ and $w_{S}$ for the absence and presence of scattering; the refractive index for medium $n$; the radius of the sample beam at the focal plane $\omega_{0}$; and the focal length of objective lens $f$, as follows:

$$
\begin{aligned}
& \left\langle S^{2}(z)\right\rangle \alpha \exp \left(-2 \mu_{\mathrm{s}} z\right)+\frac{2 \exp \left(-2 \mu_{\mathrm{s}} z\right)\left[1-\exp \left(-\mu_{\mathrm{s}} z\right)\right]}{1+\frac{W_{S}^{2}}{W_{H}^{2}}} \\
& \quad+\left[1-\exp \left(-\mu_{\mathrm{s}} z\right)\right]^{2} \frac{W_{H}^{2}}{W_{S}^{2}}, \\
& \frac{W_{S}^{2}}{W_{H}^{2}}=1+\frac{4}{3}\left(\mu_{\mathrm{s}} z\right)\left[\frac{\pi w_{0} \theta_{\mathrm{rms}}}{n f} z\right]^{2} .
\end{aligned}
$$

In the EHF theory, it should be noted that it is not easy to predict the optical coefficients of $g$ and $\mu_{\mathrm{s}}$ at the same time due to the fact that they are competing parameters. Therefore, using the other method to obtain one of them is a general solution to approach. After obtaining the $g_{\text {dep }}$ of mesoporous $\mathrm{TiO}_{2}$ beads with different diameter sizes for various IPS from the FDTD method, introducing the value of $g_{\text {dep }}$ for specific $\mathrm{TiO}_{2}$ beads samples, the corresponding scattering coefficient $\mu_{\mathrm{s}}$ can be further derived for the PSF of A-scan signal based on EHF theory.

(a)

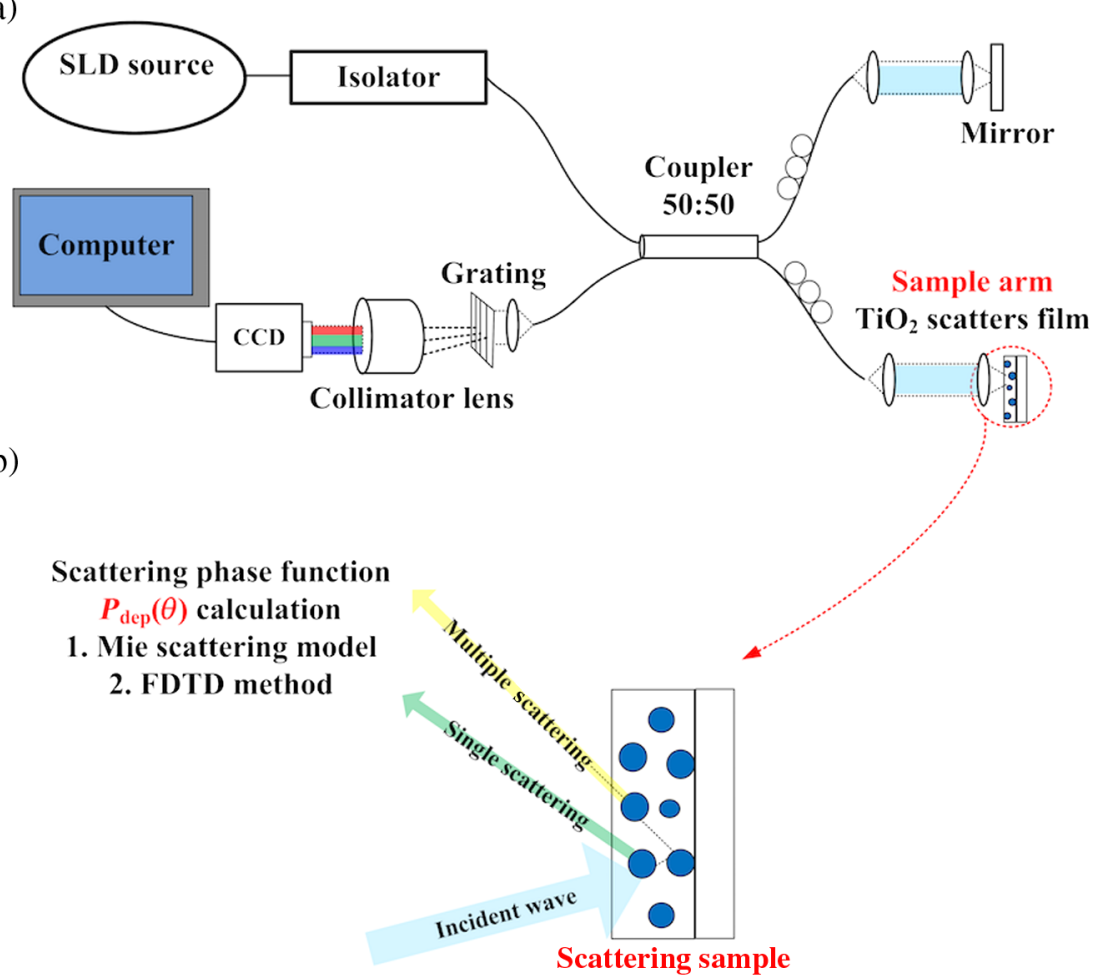

Fig. 2 (a) Schematic diagram of SD-OCT system at the central wavelength of $853 \mathrm{~nm}$, where the mesoporous $\mathrm{TiO}_{2}$ films with different average diameters are placed on the sample arm. (b) Schematic diagram of the sample shows the scattering effects of mesoporous $\mathrm{TiO}_{2}$ scatter films occur, with incident lightwave illuminated on the samples. 


\subsection{Optical Coherence Tomography Setup}

The schematic diagram of the SD-OCT system using an 853-nm super-luminescent diode as the source is shown in Fig. 2(a). A single mode fiber (Thorlabs $780 \mathrm{HP}$ ) is used to direct the light into the reference and sample arms. This OCT system has a numerical aperture of 0.042 , with an objective lens focal length of $17.9 \mathrm{~mm}$. The interferometer signal strikes a transmission grating (1200 lines/mm) and the objective lens then collects and focuses the diffracted light onto the CCD (e2V, 2048 pixels, 28-kHz line rate). Each A-scan profile (in depth) was averaged over 1000 measurements and subtracted by the reference arm intensity, to obtain the interferometer signal without the DC term.

\section{Results}

\subsection{Scattering Phase Function of Single $\mathrm{TiO}_{2} \mathrm{Bead}$ Simulated by FDTD}

The optical behavior of a single $\mathrm{TiO}_{2}$ bead with different diameters has to be confirmed prior to the simulation of multiple beads, with the FDTD method analysis. Figure 3 shows the plots of angular-dependent scattering patterns of a single $\mathrm{TiO}_{2}$ bead with diameters of 20,150,300, and $500 \mathrm{~nm}$, obtained by using the FDTD method, and is compared with the results from the Mie scattering model. ${ }^{24-26}$ These comparison results show that both methods are consistent and verify that the settings in the FDTD method are appropriate. The resultant values of $g_{\text {dep }}$ obtained for single $\mathrm{TiO}_{2}$ bead with diameters from 20, 150, 300, and $500 \mathrm{~nm}$ are $0.003,0.174,0.525$, and 0.327 , respectively.

\subsection{Interparticle Spacing Effects of Near-Field Scattering Patterns}

To study the multiple scattering effects that occur within high density tissues, multiple $\mathrm{TiO}_{2}$ beads (for smaller IPS in Sec. 2.2) are simulated, with near-field scattering patterns as shown in Fig. 4(b). In analyzing actual OCT signals, the presence of multiple scattering makes it difficult to acquire the corresponding anisotropy factors $g_{\text {dep }} \cdot{ }^{27,28}$ We simplify the scatter geometry to in-line configuration of $\mathrm{TiO}_{2}$ beads separated by different IPS in order to investigate the scattering phenomenon. In Fig. 4(a), the electrical field patterns for in-line $\mathrm{TiO}_{2}$ beads with a diameter of $300 \mathrm{~nm}$ are shown; the IPS is varied from 0 to $1000 \mathrm{~nm}$ in intervals of $250 \mathrm{~nm}$. The light propagates in the upward direction and is incident onto the beads from underneath, as shown in Fig. 4. According to the results of far-field scattering patterns, except for the main lobe that resulted from the Mie scattering effect, the presence of side-lobes was found by considering the effect of multiple scattering. This needs to be noted when the IPS is increased.
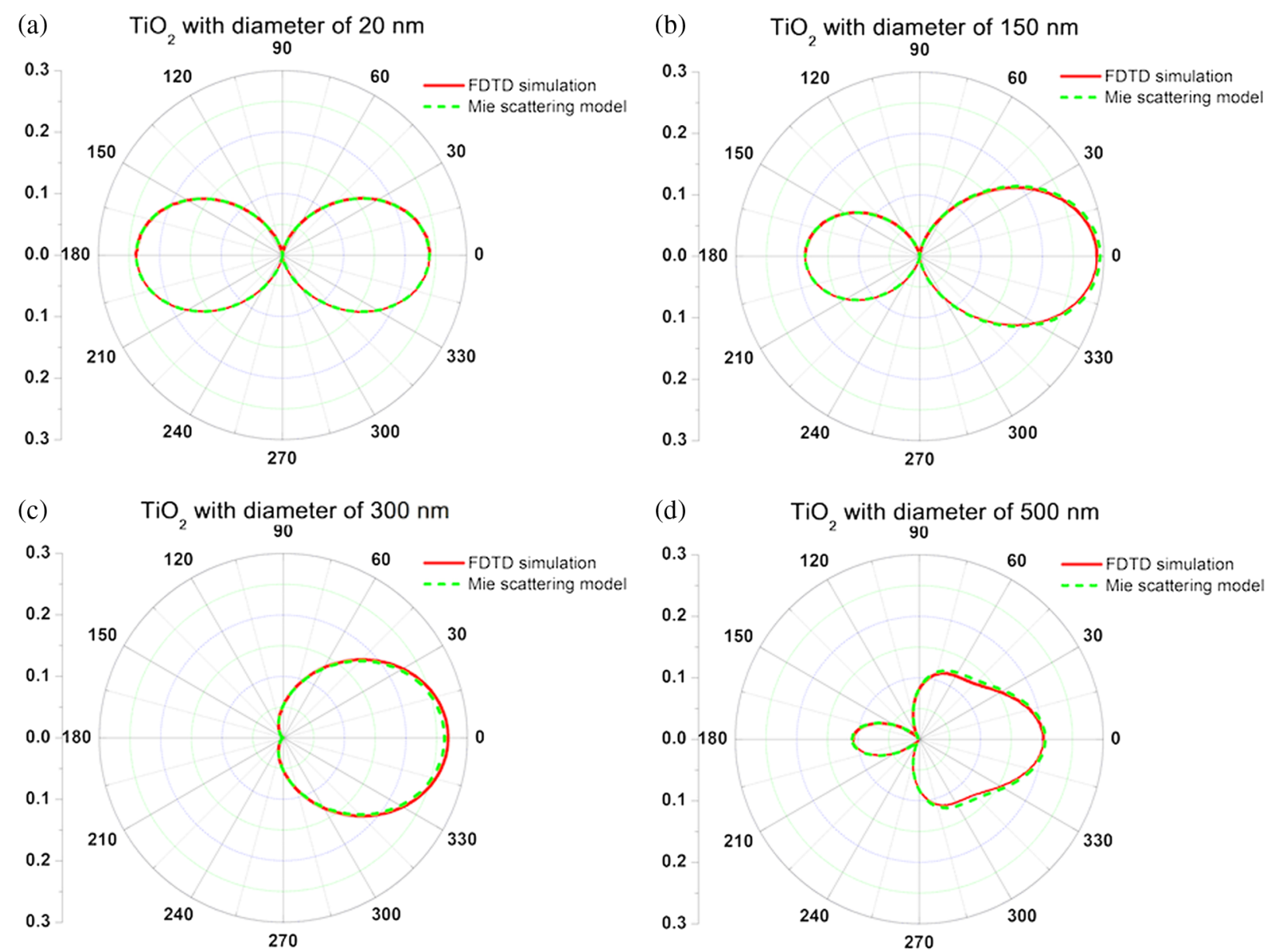

Fig. 3 Far-field scattering patterns of single $\mathrm{TiO}_{2}$ bead calculated from Mie scattering model (red solid line) and compared with FDTD method (green dash line) for different diameters of (a) 20, (b) 150, (c) 300, and (d) $500 \mathrm{~nm}$. 
Tsai et al.: Quantifying scattering coefficient for multiple scattering effect by combining optical coherence tomography...

(a) Geometric structure of $\mathrm{TiO}_{2}$ beads

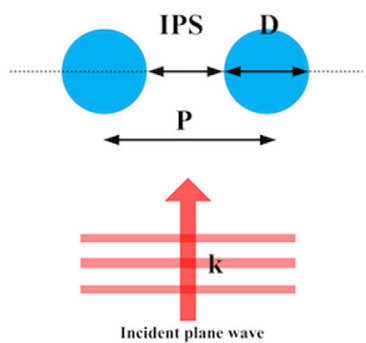

(b)

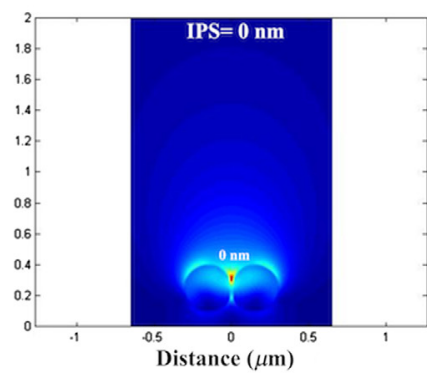

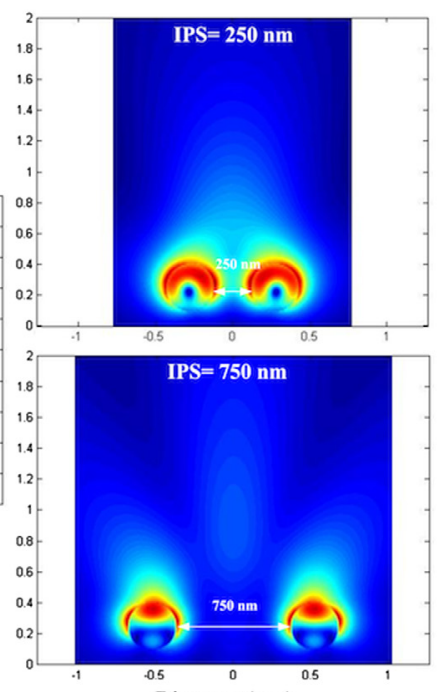

Distance $(\mu \mathrm{m})$
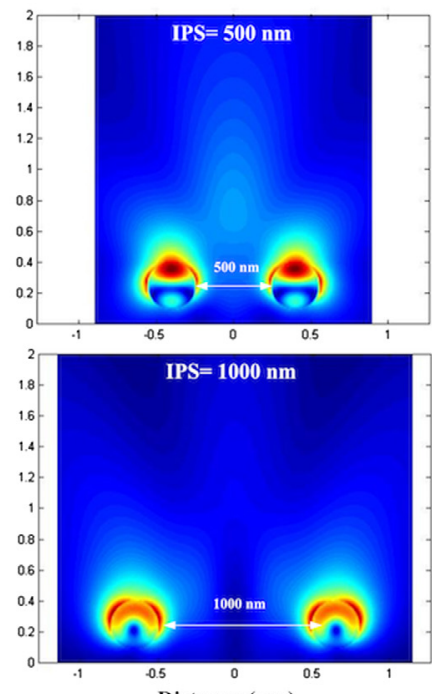

Distance $(\mu \mathrm{m})$

Fig. 4 (a) The modeling geometric structure schematic of mesoporous in-line $\mathrm{TiO}_{2}$ beads embedded in a unit cell with different diameters $(D)$ of $20,150,300$, and $500 \mathrm{~nm}$, which show the various IPS between adjacent $\mathrm{TiO}_{2}$ beads. (b) Scattering patterns of near-field electrical field magnitude of in-line $\mathrm{TiO}_{2}$ beads for $300 \mathrm{~nm}$ separated by IPS. The light is incident from the bottom in each subfigure.

(a) $\mathrm{TiO}_{2}$ with diameter of $20 \mathrm{~nm}$

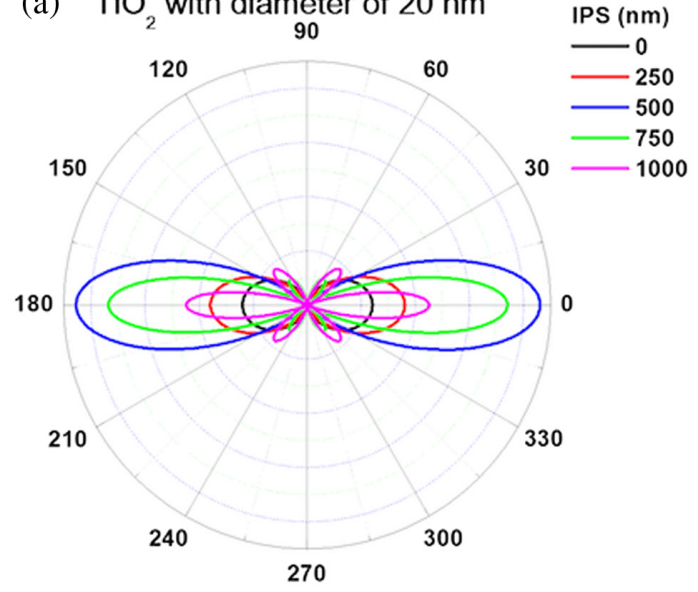

(c) $\mathrm{TiO}_{2}$ with diameter of $300 \mathrm{~nm}$

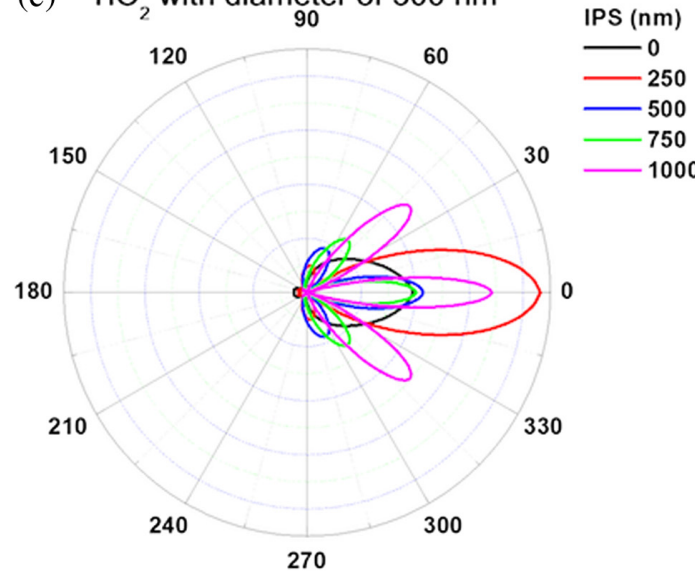

(b) $\mathrm{TiO}_{2}$ with diameter of $150 \mathrm{~nm}$

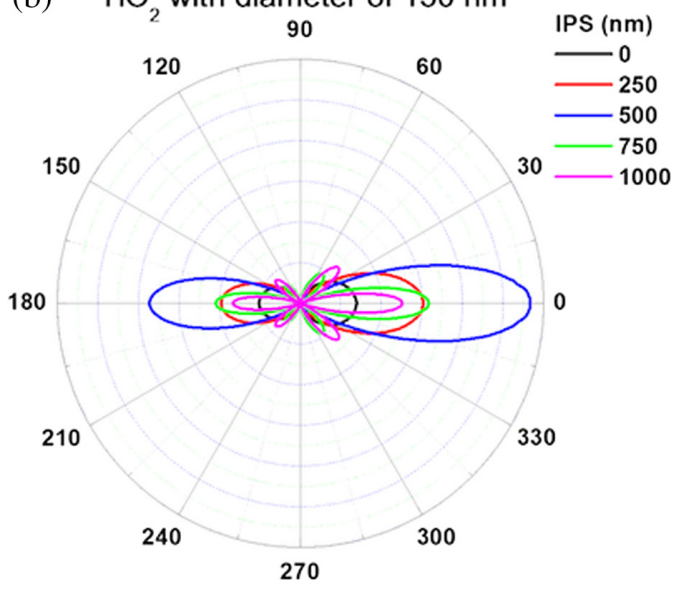

(d) $\mathrm{TiO}_{2}$ with diameter of $500 \mathrm{~nm}$

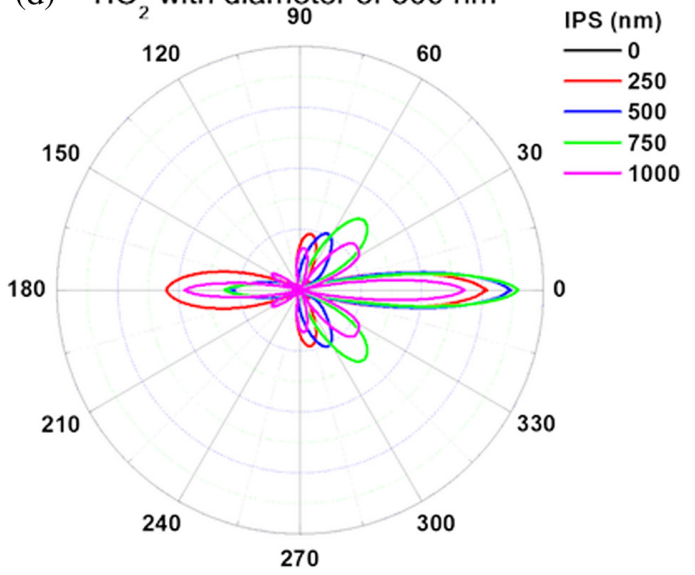

Fig. 5 Far-field angular scattering patterns [phase function $p_{\text {dep }}(\theta)$ ] against variable IPS from 0, 250, 500, 750 , and $1000 \mathrm{~nm}$ of $\mathrm{TiO}_{2}$ beads for different diameters of (a) 20, (b) 150 , (c) 300 , and (d) $500 \mathrm{~nm}$. 


\subsection{Interparticle Distance Effects-Dependent Phase Function}

To approach the realistic $g_{\text {dep }}$ [Eq. (1)], the far-field angular phase function $\left[p_{\text {dep }}(\theta)\right]$ of in-line $\mathrm{TiO}_{2}$ beads was further simulated using the FDTD method. Figure 5 shows the effect of IPS on the angular scattering patterns for mesoporous $\mathrm{TiO}_{2}$ beads with diameters of 20, 150,300, and $500 \mathrm{~nm}$. In each case, we observe adjusting the value of $P(P=\mathrm{IPS}+\mathrm{D})$, where $P$ is the period for in-line $\mathrm{TiO}_{2}$ beads, which can be defined as the sum of IPS and the diameter of $\mathrm{TiO}_{2}$ bead. Changing of $P$ induces variation on the phase function $\left[p_{\text {dep }}(\theta)\right]$ [Figs. 5(a)-5(d)], including the contribution ratio between backward and forward scatterings, and the appearance of side-lobes. Apart from the characteristic nature of Mie scattering $\left[p_{\mathrm{Mie}}(\theta)\right]$, shown for each diameter size of $\mathrm{TiO}_{2}$ bead, the incident wave also leads to scattering field interference through the propagation path.

For the 150-nm-diameter $\mathrm{TiO}_{2}$ beads, the appearance of sidelobes can be found as the IPS is increased beyond $750 \mathrm{~nm}$. When IPS is smaller than $500 \mathrm{~nm}$, the scattering patterns are similar to Mie scattering ones; these can be maintained except for the main-lobe energy leakage with increasing IPS, without diffraction associated side-lobes. The side-lobes can be detected at scattering angles of $52 \mathrm{deg}$ and $128 \mathrm{deg}$. The two lobes are symmetric with respect to the main lobe, and when reaching the condition of $P>\lambda_{0} / 2$, the interference phenomenon induced by phase delay between adjacent $\mathrm{TiO}_{2}$ beads [see Fig. 4(b)] produces a grating diffraction effect. ${ }^{29,30}$ The interference pattern can be likened to a multiple-slit interference pattern from the geometric arrangement of in-line $\mathrm{TiO}_{2}$ beads [Fig. 4(a)], by analogizing the diameter of $\mathrm{TiO}_{2}$ beads as the width of the slit, and the $\mathrm{TiO}_{2}$ bead periodicity as the grating period. The detected intensity was simulated by the function which can be expressed ${ }^{31}$ as $I=I_{0} P(\theta)\left[\frac{\sin ^{2}(\alpha)}{\alpha^{2}}\right] \cos ^{2}(\beta)$, where $I_{0}$ is the intensity of incident wave, $\alpha$ is the signal of single slit diffraction, where $\alpha=\frac{1}{2} k D \sin \theta$, and $\beta$ is the signal of the wave interference by phase delay, where $\beta=\frac{1}{2} k P \sin \theta$. The minor side-lobes can be approximated by using the above equations at the angles of $48.1 \mathrm{deg}$ and $131.4 \mathrm{deg}$ with some small, but acceptable within margin, amount of errors, as compared with the data from the FDTD method. As shown in Figs. 6(a)-6(d), the $g_{\text {dep }}$ factors of $\mathrm{TiO}_{2}$ beads with different diameters from 20 to $500 \mathrm{~nm}$ versus the IPS from 0 to $1000 \mathrm{~nm}$ were simulated and calculated by the FDTD method, which integrated the phase function overall the scattering angles. In addition, the curves as the function of IPS for these samples were fitted with the B-spline function. ${ }^{32}$ With the experimental volume fraction condition of 0.03 , the corresponding $g_{\text {dep }}$ values extracted from the curves in Figs. 6(a)-6(d) are 0.0025, 0.1685, 0.3181, and 0.3866 for $\mathrm{TiO}_{2}$ bead diameters of $20,150,300$, and
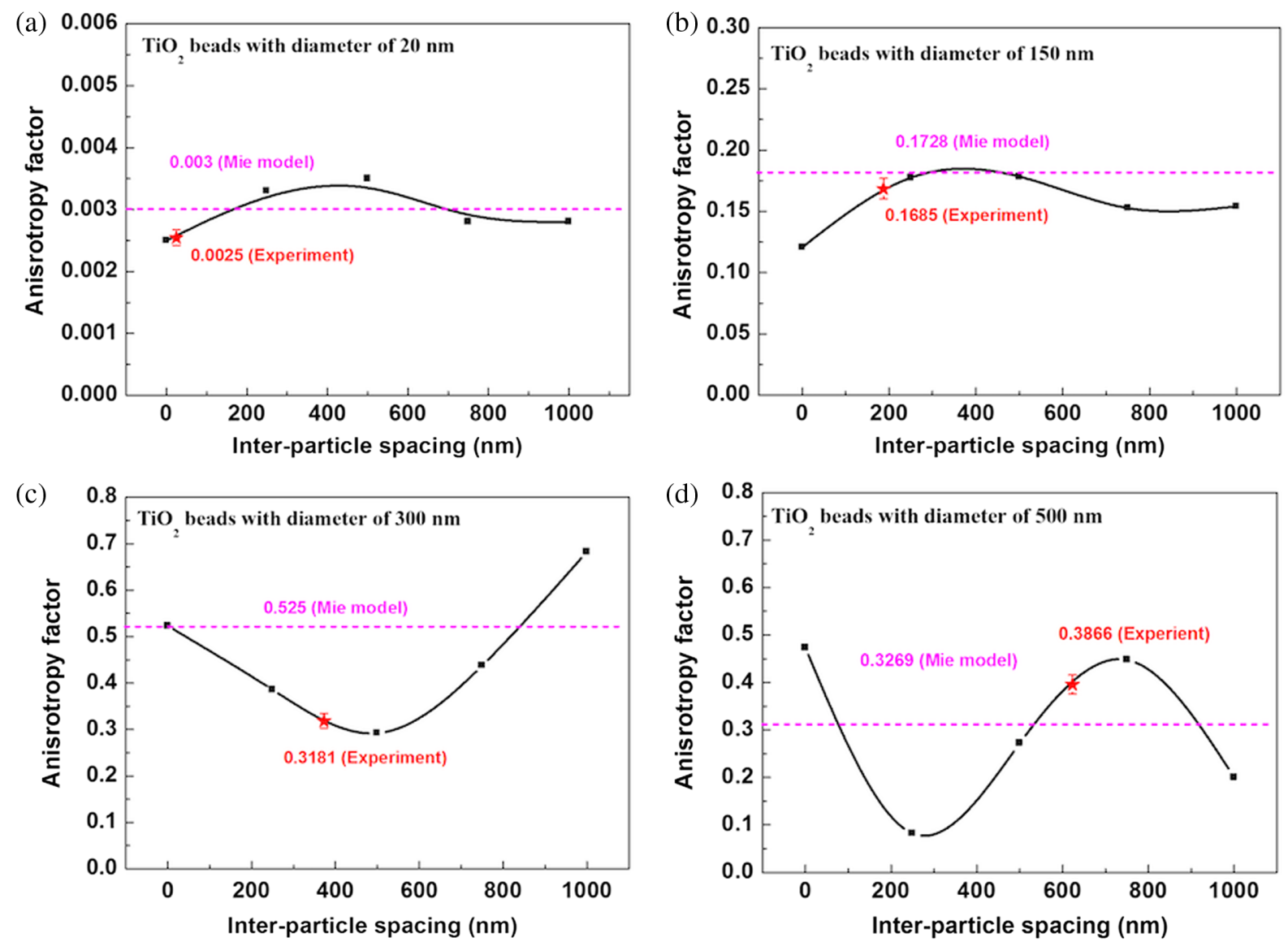

Fig. 6 The curves of simulated anisotropy factors $\left(g_{\text {dep }}\right)$ as a function of variable IPS with the point at IPS of $0,250,500,750$, and $1000 \mathrm{~nm}$ for $\mathrm{TiO}_{2}$ beads with different diameters of (a) 20, (b) 150, (c) 300, and (d) $500 \mathrm{~nm}$. The dash lines are the $g$ factors calculated based on a Mie scattering model, and the red star shows the $g$ factor of experimental condition with an error value of $5 \%$ as compared to the value on the curve. 
$500 \mathrm{~nm}$, respectively. Introducing the specific IPS values of 25 , 187, 373, and $623 \mathrm{~nm}$ for $\mathrm{TiO}_{2}$ diameters between 20 and $500 \mathrm{~nm}$ with the volume fraction of 0.03 into FDTD method, shows the slight difference within $5 \%$ error deviation as compared to the curve of $g_{\text {dep }}$ as simulated in Figs. 6(a)-6(d). Therefore, the curves of $g_{\text {dep }}$ versus IPS can be further used in scattering analysis, and the scattering coefficients $\left(\mu_{\mathrm{s}}\right)$ of different samples can be obtained by inserting the $g_{\text {dep }}$ factors into EHF model. Therefore, the trend of IPS-dependent $g_{\text {dep }}$ for different diameters of $\mathrm{TiO}_{2}$ beads can be determined. As illustrated in Figs. 6(a) and 6(b), the $\mathrm{TiO}_{2}$ with smaller diameters for 20 and $150 \mathrm{~nm}$ show $g_{\text {dep }}$ factor results similar to those from the Mie scattering effect, which implies that the variation of IPS affects weakly for smaller $\mathrm{TiO}_{2}$ beads. However, when increasing the size of $\mathrm{TiO}_{2}$ up to 300 and $500 \mathrm{~nm}$, the IPS-dependent $g_{\text {dep }}$ factor deviates from the Mie scattering effect due to the diffraction and interference phenomena between adjacent $\mathrm{TiO}_{2}$ beads. With this, an approximation of the anisotropy factor $\left(g_{\text {dep }}\right)$ of each $\mathrm{TiO}_{2}$ bead sample can be made using the OCT system, and can be further introduced into the EHF theory to find the most fitted scattering coefficient $\left(\mu_{\mathrm{s}}\right)$.

\section{Extended Huygens-Fresnel Theory}

After verifying the value of $g_{\text {dep }}$ for each of the $\mathrm{TiO}_{2}$ bead samples, the corresponding $\mu_{\mathrm{s}}$ can be further fitted by the EHF theory. Figure 7(a) shows a plot of OCT A-scans for the experimental and fitted curves of the mesoporous $\mathrm{TiO}_{2}$ sample with $300 \mathrm{~nm}$ diameter. With the fitted results, the slope of A-scan signals is dependent on the optical parameters $\left(g, \mu_{\mathrm{s}}\right)$ introduced by the EHF model, simulated by fixing the $g_{\text {dep }}$ factor from the Mie scattering model and FDTD method. In Fig. 7(b), the scattering coefficient fitted for different $\mathrm{TiO}_{2}$ bead diameters is shown. When increasing the diameter from 20 to $500 \mathrm{~nm}$, the calculated scattering coefficient is $12.9 \pm 0.3,20.8 \pm 0.3$, $14.2 \pm 0.3$, and $19.5 \pm 0.3 \mathrm{~mm}^{-1}$, with the $g_{\text {dep }}$ factors calculated from the FDTD method. In comparison, the calculated scattering coefficient is $13.5 \pm 0.6,16.5 \pm 0.6,20.2 \pm 0.6$, and $18.5 \pm 0.6 \mathrm{~mm}^{-1}$, respectively, when the $g$ factors are obtained by using the Mie scattering model. In Table 1, the calculated correlation factors $(\rho)$, between the experimental results and the fitted OCT results, show good agreement $(\rho=1)$, verifying the accuracy of the obtained IPS-dependent $\mu_{\text {s-FDTD }}$ with $g_{\text {dep }}$ factors for each $\mathrm{TiO}_{2}$ bead sample. The $\rho$ can be calculated from the mean and stand deviation of the experiment, with fitted A-scan signals to estimate the linear dependence between these two signals. ${ }^{33}$ The highest $\rho$ can be calculated for different $\mathrm{TiO}_{2}$ bead diameters of $20,150,300$, and $500 \mathrm{~nm}$, and the results are $0.9431,0.9617,0.9721$, and 0.9241 , respectively. This shows that, once the $g_{\text {dep }}$ for specific IPS can be accurately determined, the corresponding scattering coefficient $\mu_{\mathrm{s}}$ can be more accurately quantified with reduced error value from 0.6 to 0.3 . Also from the results, the $\mu_{\mathrm{s} \text {-MIE }}$ fitted can be observed to be overestimated, when the $\mathrm{TiO}_{2}$ bead diameter is increased up to $300 \mathrm{~nm}$, for which the multiple scattering effect becomes more pronounced along with the increased $\mathrm{TiO}_{2}$ bead diameter. The oscillation behavior of scattering coefficient $\mu_{\mathrm{s}}$ for different $\mathrm{TiO}_{2}$ bead diameters from 100 to $300 \mathrm{~nm}$ is shown in Table 1 and Fig. 7(b) while the fitting is based on FDTD method. Due to the limitations of the experiment, the scattering coefficient trend can only be observed within the narrow range of variable $\mathrm{TiO}_{2}$ bead sizes. However, similar results can also be found in other research. ${ }^{34}$ In addition, the $\mu_{\text {s-FDTD }}$ fit can be obtained when $g_{\text {dep }}$

Table 1 Correlation factor of scattering coefficient analysis for both FDTD method and Mie scattering model.

\begin{tabular}{lccc}
$\mathrm{TiO}_{2}$ beads $(D)$ & $\theta_{\mathrm{rms}}(\mathrm{Mie})$ & $\mu_{\mathrm{s}}(\mathrm{Mie})$ & Correlation factor \\
\hline $20 \mathrm{~nm}$ & 1.5678 & $13.5 \pm 0.6$ & 0.9429 \\
$150 \mathrm{~nm}$ & 1.3961 & $16.5 \pm 0.6$ & 0.9559 \\
$300 \mathrm{~nm}$ & 1.0183 & $20.2 \pm 0.6$ & 0.9613 \\
$500 \mathrm{~nm}$ & 1.2378 & $18.5 \pm 0.6$ & 0.9243
\end{tabular}

\begin{tabular}{lccc}
\hline $\mathrm{TiO}_{2}$ beads $(D)$ & $\theta_{\mathrm{rms}}$ (FDTD) & $\mu_{\mathrm{s}}$ (FDTD) & Correlation factor \\
\hline $20 \mathrm{~nm}$ & 1.5683 & $12.9 \pm 0.3$ & 0.9431 \\
$150 \mathrm{~nm}$ & 1.4015 & $20.8 \pm 0.3$ & 0.9617 \\
$300 \mathrm{~nm}$ & 1.2472 & $14.2 \pm 0.3$ & 0.9721 \\
$500 \mathrm{~nm}$ & 1.1739 & $19.8 \pm 0.3$ & 0.9241 \\
\hline
\end{tabular}

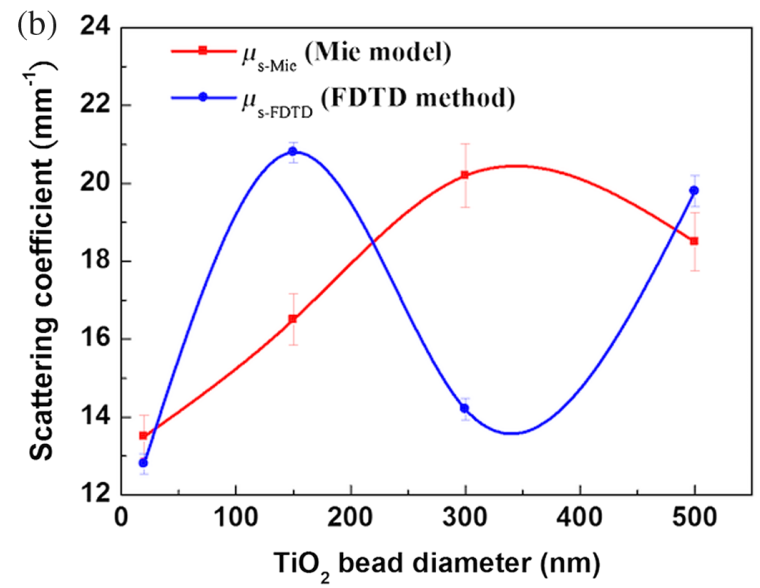

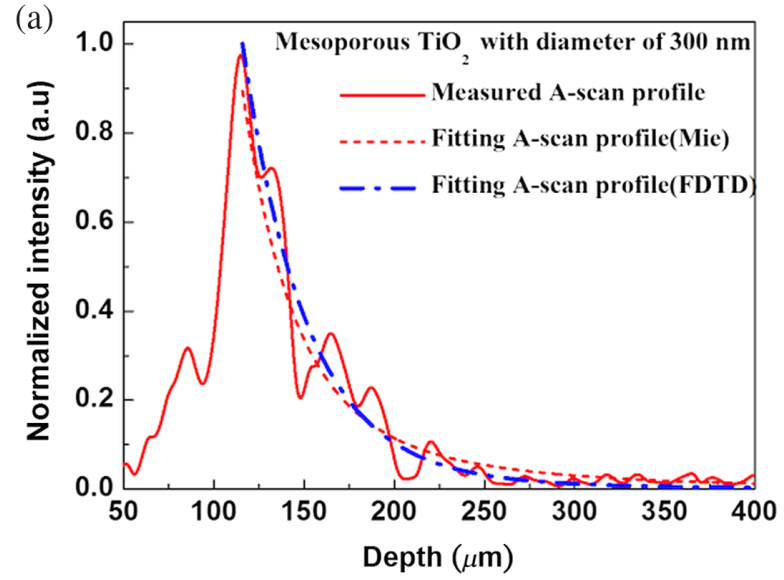

Fig. 7 (a) Experimentally measured OCT A-scan signal and the fitted A-scan signals based on Mie scattering model and FDTD method. (b) The curves of fitted scattering coefficients based on Mie model and FDTD method for different $\mathrm{TiO}_{2}$ bead diameters of 20,150,300, and $500 \mathrm{~nm}$. 
factors calculated from the FDTD method show the smaller error values decreased from 0.6 to 0.3 . The results of lower $\mu_{\text {s-FDTD }}$ as compared with $\mu_{\mathrm{s}-\mathrm{MIE}}$ are consistent with the findings from other research, ${ }^{9,11}$ which had showed that the presence of the multiple scattering effect, due to larger diameter size and larger volume fraction, can induce the deviation from the $\mu_{\mathrm{s}}$ calculated based on Mie scattering. Therefore, in these cases, the FDTD method is proved to be useful for accurately quantifying the optical properties for multiple scattering.

\section{Discussion}

Alternatively, one similar analysis was shown using the structure factor in Percus-Yevic theory (2015). ${ }^{11}$ It interpreted the spherical particles illuminated by scattering light that induce the nonlinearly scattering cross section, to show that the oscillatory behavior depends on the volume fraction. With scatter of larger diameter, despite taking into account the concentration dependence, the optical properties induced by multiple scattering cannot be completely described. Our study based on the FDTD method can be used to directly observe the far-field scattering pattern for different $\mathrm{TiO}_{2}$ samples. In addition, with methods based on Monte Carlo simulation, it is difficult to perform proper algorithm modeling. ${ }^{35,36}$ Therefore, in this work, the OCT and FDTD combination method stands out for its flexibility in simulating complex structures (cellular morphology, cancer cells, etc. $)^{37,38}$ to directly acquire the original phase function $p_{\text {dep }}(\theta)$ for further expansions in biological applications, and as opposed to making indirect predictions in the other methods mentioned above.

\section{Conclusion}

In this work, we successfully demonstrated that combining the OCT system with the FDTD method can be used to obtain the $g_{\text {dep-FDTD }}$ factor of high density tissues in the presence of multiple scatterings. Combining with the FDTD method, the light scattering interactions for near- and far-field patterns between adjacent $\mathrm{TiO}_{2}$ beads can be determined to identify the anisotropy $g$ factor, which also can be utilized for other scatters with different shapes or arrangements. The $\mu_{\text {s-FDTD }}$ can be further extracted by using a fitting process based on the EHF theory. The increase in the sample density is accompanied by smaller IPS, and the near-field scattering patterns show that the photons interact and interfere between adjacent scatters through propagating the sample. A new analysis flow to accurately quantify the optical properties of the samples through the use of OCT and FDTD combination method is flexible and has future potential in the biomedical field.

\section{Disclosures}

The authors have no relevant financial interests in this article and no conflicts of interest to disclose.

\section{Acknowledgments}

The authors gratefully acknowledge the support from the Ministry of Science and Technology of Taiwan under Contract Nos. MOST 106-2221-E-002-155-MY3, 105-2221-E-002-121, and MOST 107-3113-E-155-001-CC2 and National Taiwan University under the Aim for Top University Project 105R8908.

\section{References}

1. D. Huang et al., "Optical coherence tomography," Science 254(5033), 1178-1181 (1991).

2. A. F. Fercher, "Optical coherence tomography," J. Biomed. Opt. 1(2), 157-173 (1996).

3. W. Drexler et al., Optical Coherence Tomography: Technology and Applications, 2nd ed., Springer International Publishing, Switzerland (2015).

4. D. J. Faber et al., "Are quantitative attenuation measurements of blood by optical coherence tomography feasible?" Opt. Lett. 34(9), 14351437 (2009).

5. P. Elbau et al., "Mathematical methods of optical coherence tomography," in Handbook of Mathematical Methods in Imaging, O. Scherzer, Ed., pp. 1169-1204, Springer, New York (2015).

6. M. Santos et al., "Simulation of cellular changes on optical coherence tomography of human retina," in 37th Annual Int. Conf. of the IEEE Engineering in Medicine and Biology Society (EMBC), pp. 81478150 (2015)

7. L. Thrane et al., "Analysis of optical coherence tomography systems based on the extended Huygens-Fresnel principle," J. Opt. Soc. Am. A 17(3), 484-490 (2000).

8. V. M. Koach et al., "Determination of the scattering anisotropy with optical coherence tomography," Opt. Express 19(7), 6131-6140 (2011).

9. V. D. Nguyen et al., "Dependent and multiple scattering in transmission and backscattering optical coherence tomography," Opt. Express 21(21), 29145-29156 (2013).

10. J. K. Percus et al., "Analysis of classical statistical mechanics by means of collective coordinates," Phys. Rev. 110(1), 1-13 (1958).

11. M. Almasian et al., "Validation of quantitative attenuation and backscattering coefficient measurements by optical coherence tomography in the concentration-dependent and multiple scattering regime," J. Biomed. Opt. 20(12), 121314 (2015).

12. G. M. Conley et al., "Light transport and localization in two-dimensional correlated disorder," Phys. Rev. Lett. 112, 143901 (2014).

13. A. Taflove et al., Computational Electrodynamics: The Finite Difference Time-Domain Method, Artech House, Boston (1995).

14. J. He et al., "Light scattering by multiple red blood cells," J. Opt. Soc. Am. A 21(10), 1953-1961 (2004).

15. M. S. Starosta et al., "Far-field superposition method for three-dimensional computation of light scattering from multiple cells," J. Biomed. Opt. 15(5), 055006 (2010).

16. J. Q. Lu et al., "Simulations of light scattering from a biconcave red blood cell using the finite-difference time-domain method," $J$. Biomed. Opt. 10(2), 024022 (2005).

17. K. S. Yee et al., "Numerical solution of initial boundary value problems involving Maxwell's equations in isotropic media," IEEE Trans. Antennas Propag. 14(3), 302-307 (1966).

18. S. Hore et al., "Influence of scattering layers on efficiency of dye-sensitized solar cells," Sol. Energy Mater. Sol. Cells 90(9), 1176-1188 (2006).

19. H. I. Elim et al., "Refractive index control and Rayleigh scattering properties of transparent $\mathrm{TiO}_{2}$ nanohybrid polymer," J. Phys. Chem. B 113(30), 10143-10148 (2009).

20. J. Muller et al., "Finite-difference time-domain and near-field-to-farfield transformation in the spectral domain: application to scattering objects with complex shapes in the vicinity of a semi-infinite dielectric medium," J. Opt. Soc. Am. A 28(5), 868-878 (2011).

21. T. Hao et al., "Calculation of interparticle spacing in colloidal systems," J. Colloid Interface Sci. 297(1), 374-377 (2006).

22. A. Kienle et al., "Determination of the scattering coefficient and the anisotropy factor from laser Doppler spectra of liquids including blood," Appl. Opt. 35(19), 3404-3412 (1996).

23. J. C. Y. Kah et al., "Concentration dependence of gold nanoshells on the enhancement of optical coherence tomography images: a quantitative study," Appl. Opt. 48(10), D96-D108 (2009).

24. S. A. Prahl et al., "Mie scattering calculators," http://www.omlc.ogi.edu/ calc/miecalc.html (2006).

25. J. M. Steinke et al., "Comparison of Mie theory and the light scattering of red blood cells," Appl. Opt. 27(19), 4027-4033 (1998).

26. X. Li et al., "Modified FDTD near-to-far-field transformation for improved backscattering calculation of strongly forward-scattering objects," IEEE Antennas Wirel. Propag. Lett. 4(1), 35-38 (2005). 
27. L. E. McNeil et al., "Orientation dependence in near-field scattering from $\mathrm{TiO}_{2}$ particles," Appl. Opt. 40(22), 3726-3736 (2001).

28. J. Piskozub et al., "Effective scattering phase functions for the multiple scattering regime," Opt. Express 19(5), 4786-4794 (2011).

29. D. Sikdar et al., "Optically resonant magneto-electric cubic nanoantennas for ultra-directional light scattering," J. Appl. Phys. 117(8), 083101 (2015).

30. W. Liu et al., "Broadband unidirectional scattering by magneto-electric core shell nanoparticles," ACS Nano 6(6), 5489-5497 (2012).

31. A. Ammar et al., "Optics simulation with Python: diffraction," Proc. SPIE 9793, 97930K (2015).

32. P. Gans and J. B. Gill, "Smoothing and differentiation of spectroscopic curves using spline functions," Appl. Spectrosc. 38(3), 370-376 (1984).

33. R. A. Fisher et al., Statistical Methods for Research Workers, 13th ed., Oliver and Boyd, Edinburgh, London (1958).

34. S. Hore et al., "Scattering spherical voids in nanocrystalline $\mathrm{TiO}_{2}$ enhancement of efficiency in dye-sensitized solar cells," Chem. Commun. (15), 2011-2013 (2005).

35. B. Karamata et al., "Multiple scattering in optical coherence tomography. I. Investigation and modeling," J. Opt. Soc. Am. A 22, 1369-1379 (2005).

36. S. Malektaji et al., "Monte Carlo simulation of optical coherence tomography for turbid media with arbitrary spatial distributions" J. Biomed. Opt. 19(4), 046001 (2014).

37. S. Tanev et al., "Flow cytometry with gold nanoparticles and their clusters as scattering contrast agents: FDTD simulation of light-cell interaction," J. Biophotonics 2(8-9), 505-520 (2009).

38. E. Pickwell et al., "In vivo study of human skin using pulsed terahertz radiation," Phys. Med. Biol. 49(9), 1595-1607 (2004).

Ling-Hsuan Tsai is a PhD candidate at the Graduate Institute of Photonics and Optoelectronics, College of Electrical Engineering and Computer Science, National Taiwan University. She received her BS degree from Feng Chia University and her MS degree from the National Sun Yat-Sen University in 2008 and 2010, respectively. Her current research interests are utilizing optical coherence tomography system to analyze scattering optical properties information from tissue and further obtain accurate scattering parameters.

Po Nien Yang is a PhD candidate at the Graduate Institute of Photonics and Optoelectronics, College of Electrical Engineering and Computer Science, National Taiwan University. He received his BS degree from I-Shuo University and his MS degree from National Taiwan Normal University. His research interests include using optical coherence tomography systems to study the physics of photon scattering, theoretical high energy physics phenomenology, and quantum field theory.

Chung-Chih Wu received his PhD from Princeton University in 1997. Currently, he is a distinguished professor of Department of Electrical Engineering, Graduate Institute of Photonics and optoelectronics, and Graduate Institute of Electronics Engineering, National Taiwan University.

Hoang Yan Lin received his BS and PhD degrees from the Electrical Engineering Department and the Graduate Institute of Electrical Engineering at National Taiwan University in 1987 and 1993, respectively. His research interests are design of optical components and integration of optical systems. He is the representative for the Taipei section of the Institute of Electronics, Information, and Communication Engineers, and a member of the Optical Society of America, the Society for Information Display, and the 3D Interaction \& Display Association. 